Internat. J. Math. \& Math. Sci.

Vol. 23, No. 9 (2000) 605-616

S0161171200001897

(C) Hindawi Publishing Corp.

\title{
A GLOBAL METHOD FOR SOME CLASS OF OPTIMIZATION AND CONTROL PROBLEMS
}

\author{
R. ENKHBAT
}

(Received 6 August 1998)

\begin{abstract}
The problem of maximizing a nonsmooth convex function over an arbitrary set is considered. Based on the optimality condition obtained by Strekalovsky in 1987 an algorithm for solving the problem is proposed. We show that the algorithm can be applied to the nonconvex optimal control problem as well. We illustrate the method by describing some computational experiments performed on a few nonconvex optimal control problems.
\end{abstract}

Keywords and phrases. Global optimization, optimal control, optimality condition, convex function, simple set.

2000 Mathematics Subject Classification. Primary 90C26; Secondary 65K05, 90C25, 90C55.

1. Introduction. In the paper [2], we developed an algorithm for maximizing a differentiable convex function on a so-called simple set. Continuing this work, we give such a method for maximizing a nondifferentiable convex function, and also for maximizing a nonconvex optimal control problem.

This paper is organized as follows. In Section 2, we consider the global optimality condition [13] for the problem of maximizing a convex function. In Sections 3 and 4, we construct a method based on the global optimality condition for solving the problem and show convergence of the algorithm. In Section 6, we apply the proposed algorithm to the nonconvex optimal control problem for a terminal functional. In Section 7, we present some computational results obtained with our algorithm on a few optimal control test problems.

2. Global optimality condition. We consider the problem

$$
f(x) \longrightarrow \max , \quad x \in D \subset \mathbb{R}^{n},
$$

where $f: \mathbb{R}^{n} \rightarrow \mathbb{R}$ is a convex function and $D$ is an arbitrary subset of $\mathbb{R}^{n}$. This problem belongs to the class of global optimization problems. Any local maximizer found by well-known local search methods might be differ from the global maximizer (a solution) of the problem. There are many numerical methods [2, 4, 5, 6, 9, 10, 12] devoted to the solution of problem (2.1). The global optimality conditions for problem (2.1) were first given by Strekalovsky [13]. For future purposes, let us consider this result applied to a finite dimensional space of $\mathbb{R}^{n}$.

THEOREM 2.1 [13]. If a point $z \in D$ is a global solution of problem (2.1) then the following condition holds: 


$$
\forall y: f(y)=f(z), \forall y^{*} \in \partial f(y), \quad\left\langle y^{*}, x-y\right\rangle \leq 0, \quad \forall x \in D .
$$

If, in addition to (2.2), the condition

$$
\exists v \in \mathbb{R}^{n}: f(v)<f(z)<\infty
$$

is satisfied, then condition (2.2) becomes sufficient. (Here and in the following $\langle$,$\rangle de-$ notes the scalar product of two vectors.)

Proof. Necessity. Assume that $z$ is a solution of problem (2.1). Let the points $y, y^{*}$ and $x$ be such that

$$
\begin{gathered}
y \in \mathbb{R}^{n}: f(y)=f(z), \\
y^{*} \in \partial f(y)=\left\{c \in \mathbb{R}^{n} \mid f(x)-f(y) \geq\langle c, x-y\rangle, \forall x \in \mathbb{R}^{n}\right\}, \quad x \in D .
\end{gathered}
$$

Then, by the convexity of $f$, we have

$$
0 \geq f(x)-f(z)=f(x)-f(y) \geq\left\langle y^{*}, x-y\right\rangle .
$$

SUFFICIENCY. In order to derive a contradiction, suppose that $z$ is not a solution of problem (2.1), i.e.,

$$
\exists u \in D: f(u)>f(z) .
$$

Now we introduce the closed and convex set:

$$
L(f, z)=\left\{x \in \mathbb{R}^{n} \mid f(x) \leq f(z)\right\} .
$$

Note that $\operatorname{int} L(f, z) \neq \varnothing$ and $u \notin L(f, z)$. Then there exists the projection of the point $u$ on $L(f, z)$, i.e.,

$$
\exists y \in L(f, z):\|y-u\|=\inf _{x \in L(f, z)}\|x-u\|
$$

It is obvious that

$$
\|y-u\|>0
$$

Taking into account (2.8), we conclude that the point $y$ is characterized as a solution of the following quadratic programming problem.

$$
g(x)=\frac{1}{2}\|x-u\|^{2} \longrightarrow \min , \quad x \in L(f, z) .
$$

Then the optimality condition for this problem at the point $y$ is as follows:

$$
\begin{gathered}
\exists \lambda_{0} \geq 0, \lambda \geq 0, \quad \lambda_{0}+\lambda>0, \\
\exists y^{*} \in \partial f(y), \quad \lambda_{0} g^{\prime}(y)+\lambda y^{*}=0, \quad \lambda(f(y)-f(z))=0 .
\end{gathered}
$$

Now, we show that $\lambda_{0}>0$ and $\lambda>0$. If $\lambda_{0}=0$, then (2.11) implies that $f(y)=f(z)$ and $0 \in \partial f(y)$. This contradicts (2.3). The case $\lambda=0$ is also impossible because of 
$g^{\prime}(y) \triangleq y-u=0$ which contradicts (2.9). Thus, we can put $\lambda_{0}=1$ and write (2.11) as follows:

$$
y-u+\lambda y^{*}=0, \quad \lambda>0, \quad f(y)=f(z) .
$$

Now, using this condition, we easily get

$$
\left\langle y^{*}, u-y\right\rangle=\frac{1}{\lambda}\|u-y\|^{2}>0
$$

which contradicts (2.2). This contradiction implies that the assumption that $z$ is not a solution of problem (2.1) must be false. This completes the proof.

3. Construction of a method. In order to use the global optimality condition (2.2) efficiently for solving problem (2.1) numerically, we need to make some further assumptions about the problem. These assumptions in problem (2.1) are:

(a) The objective function $f: \mathbb{R}^{n} \rightarrow \mathbb{R}$ is a strongly convex.

(b) The feasible set $D$ is a simple.

Now, let us recall the definition of a so-called simple set.

Definition 3.1 [2]. A set $D$ is a simple set if it satisfies the following conditions:

(a) $D$ is compact.

(b) The problem of maximizing a linear function on $D$ is solvable with a "Simple method". We say that a method is simple if it involves the use of the simplex method or the use of a method that gives an analytical solution to the problem of maximizing a linear function.

Throughout the rest of this paper we consider problem (2.1) under assumptions (a) and (b). Let $f^{*}$ denotes a global maximum of problem (2.1), i.e., $f^{*}=\max _{x \in D} f(x)$. We now define the auxiliary function $\pi(y)$ by

$$
\pi(y)=\max _{y^{*} \in \partial f(y)} \max _{x \in D}\left\langle y^{*}, x-y\right\rangle, \quad \forall y \in \mathbb{R}^{n} .
$$

It is well known that $\partial f(y)$ is convex and compact [16]. Let us introduce the function defined by

$$
\Theta(x)=\max _{f(y)=f(x)} \pi(y) .
$$

Using $\Theta(x)$, we can reformulate Theorem 2.1 in the following way.

THEOREM 3.2. Let an arbitrary point $x^{0} \in D$ be such that $x^{0} \neq \arg \min _{x \in \mathbb{R}^{n}} f(x)$. If $\Theta\left(x^{0}\right) \leq 0$ then this point is a solution of problem (2.1).

Proof. The proof is an obvious consequence of the inequality:

$$
\left\langle y^{*}, x-y\right\rangle=\max _{x \in D}\left\langle y^{*}, x-y\right\rangle \leq \max _{y^{*} \in \partial f(y)} \max _{x \in D}\left\langle y^{*}, x-y\right\rangle \leq \Theta\left(x^{0}\right) \leq 0
$$

which holds for all $x$ and $y, y^{*}$ such that

$$
x \in D, \quad f(y)=f\left(x^{0}\right), \quad y^{*} \in \partial f(y) .
$$


We also note that $0 \notin \partial f(y)$, therefore by Theorem 2.1, we see that $x^{0}$ is a solution of problem (2.1) and the proof is complete. Theorem 3.2 is used to verify the optimality condition (2.2).

THEOREM 3.3. Let a sequence $\left\{x^{k}\right\} \subset \mathbb{R}^{n}$ be such that

$$
f\left(x^{k}\right)>f\left(x^{k-1}\right)>\cdots>f\left(x^{0}\right), \quad x^{0} \neq \arg \min _{x \in \mathbb{R}^{n}} f(x) .
$$

Then $\exists \delta>0:\left\|y^{k}\right\| \geq \delta$ for each $y^{k} \in \partial f\left(x^{k}\right), k=0,1,2, \ldots$.

Proof. Since $f$ is strongly convex on $\mathbb{R}^{n}$ there exists a positive constant $\gamma$ such that the following inequality holds for all $x, y \in \mathbb{R}^{n}$ and for all $\alpha \in[0,1]$ :

$$
f(\alpha x+(1-\alpha) y) \leq \alpha f(x)+(1-\alpha) f(y)-\alpha(1-\alpha) \gamma\|x-y\|^{2} .
$$

Now let $x_{*}$ be a global minimizer of $f(x)$ on $\mathbb{R}^{n}$, i.e., $f\left(x_{*}\right)=\min _{x \in \mathbb{R}^{n}} f(x)$. It is well known that $x_{*}$ is unique. Then it is clear that $x^{k} \neq x_{*}$ for each $k=0,1,2, \ldots$. From the convexity of $f$ we have

$$
f(x)-f(y) \leq\langle\tilde{y}, x-y\rangle, \quad \forall x, y \in \mathbb{R}^{n}, \tilde{y} \in \partial f(x),
$$

if we substitute $x=x^{k}, y=x_{*}$ and $\alpha=1 / 2$ into formulas (3.6) and (3.7), we obtain

$$
\begin{aligned}
\frac{1}{4} \gamma\left\|x^{k}-x_{*}\right\|^{2} & \leq \frac{1}{2}\left(f\left(x^{k}\right)-f\left(\frac{1}{2} x^{k}+\frac{1}{2} x_{*}\right)\right)+\frac{1}{2}\left(f\left(x_{*}\right)-f\left(\frac{1}{2} x^{k}+\frac{1}{2} x_{*}\right)\right) \\
& \leq \frac{1}{4}\left\langle y^{k}, x^{k}-x_{*}\right\rangle+\frac{1}{4}\left\langle\tilde{y}^{*}, x_{*}-x^{k}\right\rangle \\
& =\frac{1}{4}\left\langle y^{k}-\tilde{y}^{*}, x^{k}-x_{*}\right\rangle
\end{aligned}
$$

for all $y^{k} \in \partial f\left(x^{k}\right)$ and $\tilde{y}^{*} \in \partial f\left(x_{*}\right)$. On the other hand, since $0 \in \partial f\left(x_{*}\right)$, we have

$$
\gamma\left\|x^{k}-x_{*}\right\|^{2} \leq\left\langle y^{k}, x^{k}-x_{*}\right\rangle \leq\left\|y^{k}\right\|\left\|x^{k}-x_{*}\right\| .
$$

Thus we get

$$
\gamma\left\|x^{k}-x_{*}\right\| \leq\left\|y^{k}\right\|
$$

for each $k=0,1,2, \ldots$ Moreover, by using formulas (3.7) and (3.10), we have

$$
0<f\left(x^{k}\right)-f\left(x_{*}\right) \leq\left\langle y^{k}, x^{k}-x_{*}\right\rangle \leq\left\|y^{k}\right\|\left\|x^{k}-x_{*}\right\| \leq \frac{1}{\gamma}\left\|y^{k}\right\|^{2}
$$

for each $k=0,1,2, \ldots$.

Since the sequence $\left\{f\left(x^{k}\right)\right\}$ is strictly monotonically increasing, we have

$$
0<\gamma\left(f\left(x^{0}\right)-f\left(x_{*}\right)\right) \leq\left\|y^{k}\right\|^{2} \text { for each } k=0,1,2, \ldots .
$$

Consequently, choosing $\delta=\left(\gamma\left(f\left(x^{0}\right)-f\left(x_{*}\right)\right)\right)^{1 / 2}$ the assertion is proven. 


\section{Convergence of the algorithm}

\section{ALgORITHM 4.1}

STEP 1. Choose $x^{0} \in D$ such that $x^{0} \neq \arg \min _{x \in \mathbb{R}^{n}} f(x)$.

STEP 2. To determine the value of $\Theta\left(x^{k}\right)$ solve the constrained global maximization problem

$$
\pi(y) \rightarrow \max , \quad f(y)=f\left(x^{k}\right)
$$

Let $y_{k}$ be a solution of this problem, i.e.,

$$
\Theta\left(x^{k}\right)=\pi\left(y_{k}\right)=\max _{v \in \partial f\left(y_{k}\right)} \max _{x \in D}\left\langle v, x-y_{k}\right\rangle .
$$

Also suppose that $v^{k}$ is a solution of the following problem

$$
\psi(v)=\max _{x \in D}\left\langle v, x-y_{k}\right\rangle \rightarrow \max , \quad v \in \partial f\left(y_{k}\right) .
$$

Then we have $\pi\left(y_{k}\right)=\psi\left(v^{k}\right)=\max _{x \in D}\left\langle v^{k}, x-y_{k}\right\rangle$.

The point $x^{k+1}$ can be considered as a solution of the problem:

$$
\left\langle v^{k}, x\right\rangle \longrightarrow \max , \quad x \in D
$$

It is clear that $\Theta\left(x^{k}\right)=\pi\left(y_{k}\right)=\psi\left(v^{k}\right)=\alpha_{k}$, where $\alpha_{k}=\left\langle v^{k}, x^{k+1}-y_{k}\right\rangle$.

STEP 3. If $\Theta\left(x^{k}\right) \leq 0$ then set $x^{*}=x^{k}$ and stop, $x^{*}$ is a solution.

STEP 4. Otherwise, set $k=k+1$, and go to Step 2 .

Now we show that this algorithm converges to a global maximum of problem (2.1).

THEOREM 4.2. The sequence of points $\left\{x^{k}\right\}$ produced by the above algorithm is a maximizing sequence of problem (2.1), i.e.,

$$
\lim _{k \rightarrow \infty} f\left(x^{k}\right)=f^{*}
$$

and all the limit points of the sequence $\left\{x^{k}\right\}$ are global maximizers of problem (2.1).

Proof. Note that from the construction of $\left\{x^{k}\right\}$ we have $x^{k} \in D$ and $f\left(x^{k}\right) \leq f^{*}$ for each $k=0,1,2, \ldots$. Without loss of generality, assume

$$
\Theta\left(x^{k}\right)>0 \text { for all } k=0,1,2, \ldots
$$

In fact, otherwise, there exists $k$ such that $\Theta\left(x^{k}\right) \leq 0$. Then, by Theorem 3.2, we can conclude that $x^{k}$ is a solution of problem (2.1) and the proof is complete.

Suppose, on the contrary, that $\left\{x^{k}\right\}$ is not a maximizing sequence of problem (2.1), i.e.,

$$
\lim _{k \rightarrow \infty} \sup f\left(x^{k}\right)<f^{*}=f\left(x^{*}\right),
$$

where $x^{*}$ is a global maximizer of problem (2.1). 
First, we show that the sequence $\left\{f\left(x^{k}\right)\right\}$ is strictly monotonically increasing. By the definition of $\Theta\left(x^{k}\right)$, we have

$$
\Theta\left(x^{k}\right)=\pi\left(y_{k}\right)=\left\langle v^{k}, x^{k+1}-y_{k}\right\rangle>0,
$$

where $v^{k} \in \partial f\left(y_{k}\right), f\left(y_{k}\right)=f\left(x^{k}\right), \pi\left(y_{k}\right)=\psi\left(v^{k}\right)$.

By the convexity of $f$, this implies that

$$
f\left(x^{k+1}\right)-f\left(x^{k}\right)=f\left(x^{k+1}\right)-f\left(y_{k}\right) \geq\left\langle v^{k}, x^{k+1}-y_{k}\right\rangle>0 .
$$

Hence, we obtain $f\left(x^{k+1}\right)>f\left(x^{k}\right)$, for all $k=0,1,2, \ldots$. Because the sequence $\left\{f\left(x^{k}\right)\right\}$ is bounded by the value of $f^{*}$, there exists a limit $A$, i.e., $\lim _{k \rightarrow \infty} f\left(x^{k}\right)=A$. Then recalling (4.8) and (4.9), we obtain $\lim _{k \rightarrow \infty} \Theta\left(x^{k}\right)=0$.

Now we introduce the closed and convex sets $L_{k}\left(f, x^{k}\right)=\left\{x \in \mathbb{R}^{n} \mid f(x) \leq f\left(x^{k}\right)\right\}$ for all $k=0,1,2, \ldots$. It is clear that $x^{*} \notin L_{k}\left(f, x^{k}\right)$. Then there exists the projection of the point $x^{*}$ on $L_{k}\left(f, x^{k}\right)$ such that

$$
\begin{gathered}
\exists u^{k} \in L_{k}\left(f, x^{k}\right):\left\|u^{k}-x^{*}\right\|=\inf _{x \in L_{k}\left(f, x^{k}\right)}\left\|x-x^{*}\right\|, \quad f\left(x^{k}\right)=f\left(u^{k}\right), \\
\left\|u^{k}-x^{*}\right\|>0 .
\end{gathered}
$$

Moreover, $u^{k}$ can be considered as a solution of the convex programming problem

$$
g(x)=\frac{1}{2}\left\|x-x^{*}\right\|^{2} \rightarrow \min , \quad x \in L_{k}\left(f, x^{k}\right) .
$$

Then the optimality condition for this problem at the point $u^{k}$ is

$$
\begin{gathered}
\exists \lambda_{0} \geq 0, \lambda_{k} \geq 0, \quad \lambda_{0}+\lambda_{k} \neq 0, \\
\exists y^{k} \in \partial f\left(u^{k}\right), \quad \lambda_{0} g^{\prime}\left(u^{k}\right)+\lambda_{k} y^{k}=0
\end{gathered}
$$

for all $k=0,1,2, \ldots$. Now, we show that $\lambda_{0} \neq 0$ and $\lambda_{k} \neq 0$. In fact, if $\lambda_{0}=0$, then by (4.12), it follows that $\lambda_{k}>0$ and $y^{k}=0, f\left(u^{k}\right)=f\left(x^{k}\right)$. This contradicts the fact that $x^{k} \neq \arg \min _{x \in \mathbb{R}^{n}} f(x)$ for each $k=0,1,2, \ldots$. Analogously, we show that $\lambda_{k}>0$. Since $g^{\prime}\left(u^{k}\right)=u^{k}-x^{*} \neq 0$, the case $\lambda_{k}=0$ is also impossible and we can put $\lambda_{0}=1$ and $\lambda_{k}>0$. Then we can write (4.12) as follows:

$$
u^{k}-x^{*}+\lambda_{k} y^{k}=0 .
$$

Thus, we have

$$
\lambda_{k}=\frac{\left\|u^{k}-x^{*}\right\|}{\left\|y^{k}\right\|} .
$$

On the other hand, from the definition of $\Theta\left(x^{k}\right)$, it follows that

$$
\left\langle y^{k}, x^{*}-u^{k}\right\rangle \leq \Theta\left(x^{k}\right) .
$$

Using (4.13), (4.14), and (4.15), we have

$$
\left\|y^{k}\right\|\left\|u^{k}-x^{*}\right\| \leq \Theta\left(x^{k}\right) .
$$


From the construction of $\left\{x^{k}\right\}$ the sequence $u^{k}$ is such that $f\left(x^{k}\right)=f\left(u^{k}\right)$ and $f\left(u^{k}\right)>f\left(u^{k-1}\right)$ for all $k=0,1,2, \ldots$. Then, by Theorem 3.3, we obtain $\exists \delta>0:\left\|y^{k}\right\| \geq$ $\delta$ for all $y^{k} \in \partial f\left(u^{k}\right), k=0,1,2, \ldots$. From (4.16), it follows that $0 \leq \delta\left\|u^{k}-x^{*}\right\| \leq$ $\Theta\left(x^{k}\right)$. Taking into account that $\lim _{k \rightarrow \infty} \Theta\left(x^{k}\right)=0$, we have $\lim _{k \rightarrow \infty} u^{k}=x^{*}$. By the continuity of $f$ on $\mathbb{R}^{n}$ we conclude that

$$
\lim _{k \rightarrow \infty} f\left(x^{k}\right)=\lim _{k \rightarrow \infty} f\left(u^{k}\right)=f\left(x^{*}\right) .
$$

This contradicts (4.7). This contradiction implies that the assumption that $\left\{x^{k}\right\}$ is not a maximizing sequence of problem (2.1) is false. Since $D$ is a compact set, there exists a convergent subsequence which we relabel $\left\{x^{k}\right\}$ such that $\lim _{k \rightarrow \infty} x^{k}=\tilde{x}$. By (4.17), we obtain

$$
\lim _{k \rightarrow \infty} f\left(x^{k}\right)=f(\tilde{x})=f^{*}
$$

which completes a proof of the theorem.

NotE. If $f: \mathbb{R}^{n} \rightarrow \mathbb{R}$ is a differentiable convex function, then the function $\pi(y)$ is defined as follows:

$$
\pi(y)=\max _{x \in D}\left\langle f^{\prime}(y), x-y\right\rangle, \quad \forall y \in \mathbb{R}^{n} .
$$

In addition, if $f$ is a twice differentiable, then there exists the directional derivative of $\pi(y)$ at $y$ in the direction $h \in \mathbb{R}^{n}$ which is given by the following formula [2]:

$$
\frac{\partial \pi(y)}{\partial h}=\left\langle f^{\prime \prime}(y) h, z\right\rangle-\left\langle f^{\prime \prime}(y) y+f^{\prime}(y), h\right\rangle,
$$

where $z$ is such that

$$
\left\langle f^{\prime}(y), z\right\rangle=\max _{x \in D}\left\langle f^{\prime}(y), x\right\rangle .
$$

In this case, Algorithm 4.1 is transformed into Algorithm 4.3 (see [2]) which has been implemented numerically.

\section{Algorithm 4.3}

STEP 1. Choose $x^{0} \in D$ such that $x^{0} \neq \arg \min _{x \in \mathbb{R}^{n}} f(x)$. Set $k=0$.

STEP 2. Solve the problem

$$
\pi(y) \longrightarrow \max , \quad f(y)=f\left(x^{k}\right) .
$$

Let $y_{k}$ be a solution of this problem, i.e.,

$$
\Theta\left(x^{k}\right)=\pi\left(y_{k}\right)=\max _{x \in D}\left\langle f^{\prime}\left(y_{k}\right), x-y_{k}\right\rangle
$$

and $x^{k+1}$ be a solution of the problem

$$
\left\langle f^{\prime}\left(y_{k}\right), x\right\rangle \longrightarrow \max , \quad x \in D .
$$

STEP 3. If $\Theta\left(x^{k}\right) \leq 0$ then $x^{*}=x^{k}$ and stop.

STEP 4. Otherwise, set $k:=k+1$, and go to Step 2 . 
5. Nonconvex optimal control problem. Consider the following optimal control problem:

$$
\begin{gathered}
J(u)=\varphi\left(x\left(t_{1}\right)\right) \rightarrow \max , \\
\dot{x}(t)=A(t) x+B(t) u(t)+C(t), \quad x\left(t_{0}\right)=x^{0}, \\
u \in V=\left\{u \in L_{2}^{(r)}(T) \mid u(t) \in U, t \in T\right\},
\end{gathered}
$$

where $t \in T=\left[t_{0}, t_{1}\right],-\infty<t_{0}<t_{1}<+\infty, u(t)=\left(u_{1}(t), u_{2}(t), \ldots, u_{r}(t)\right)^{T}, x(t)=$ $\left(x_{1}(t), x_{2}(t), \ldots, x_{n}(t)\right)^{T}$. Here $x^{0} \in \mathbb{R}^{n}$ is an initial state, $t_{0}, t_{1}$, and $x^{0}$ are given. Matrix functions $A(t), B(t)$, and $C(t)$ are piecewise continuous on $\left[t_{0}, t_{1}\right]$ with dimensions $(n \times n),(n \times r)$, and $(n \times 1)$, respectively. $U \subset \mathbb{R}^{r}$ is a simple set, $\varphi: \mathbb{R}^{n} \rightarrow \mathbb{R}$ is a strongly convex and differentiable function.

The problem (5.1), (5.2), and (5.3) belongs to the class of nonconvex optimal control problem and application of Pontryagin's maximum principle $[7,11]$ can give only stationary processes $(x(\cdot), u(\cdot))$. There are a number of numerical methods based on the sufficient optimality conditions of dynamic programming [1, 16] and Krotov's condition [8] devoted to nonconvex optimal control problems. Based on optimality conditions (2.2) a global optimal control search " $\mathbb{R}$ "-algorithm using a so-called "resolving set" was proposed in [15].

We show how to apply Algorithm 4.1 or Algorithm 4.3 to the solution of problem (5.1), (5.2), and (5.3). It is well known that every admissible control $u \in V$ corresponds to a unique solution $x(t)=x(t, u)$ of the Cauchy problem (5.2) by the formula [16, 17]:

$$
x(t, u)=F(t) x^{0}+\int_{t_{0}}^{t} F(t) F^{-1}(\xi)(B(\xi) u(\xi)+C(\xi)) d \xi,
$$

where $F(t)$ is a fundamental matrix with dimension $(n \times n)$ that satisfies the matrix equation

$$
\dot{F}=A(t) F, \quad F\left(t_{0}\right)=E
$$

on $T$ with the matrix $E$. Note that a state $x(t, u)$ is an absolutely continuous vectorfunction of time $t$, which satisfies the system (5.2) almost everywhere in $T$ [16]. Let us denote by

$$
\tilde{D}=\tilde{D}\left(t_{1}\right)=\left\{y \in \mathbb{R}^{n} \mid y=x\left(t_{1}, u\right), u \in V\right\}
$$

the reachable set of the control system (5.2) and (5.3). Under the above assumptions the set $\tilde{D} \subset \mathbb{R}^{n}$ is convex and compact $[11,17]$. Then we can present problem (5.1), (5.2), and (5.3) in the following form:

$$
\varphi(x) \longrightarrow \max , \quad x \in \tilde{D} .
$$

Let a point $x^{*}$ be a solution of problem (5.7), then the admissible control $u^{*}=u^{*}(t)$, $t \in T$ corresponding to $x^{*}$ is the global optimal control solution to problem (5.1), (5.2), and (5.3). Therefore, Algorithms 4.1 and 4.3 for solving problem (2.1) can also be used 
for solving the problem (5.1), (5.2), and (5.3). As we have done before, write down the auxiliary function $\pi(y)$ for problem (5.7) as follows:

$$
\tilde{\pi}(y)=\max _{x \in \tilde{D}}\left\langle\varphi^{\prime}(y), x-y\right\rangle .
$$

We first consider the following linearized optimal control problem

$$
\left\langle\varphi^{\prime}(y), x\right\rangle \rightarrow \max , \quad x \in \tilde{D} .
$$

In order to solve problem (5.9), we introduce a conjugate state

$$
\psi(t, y)=\left(\psi_{1}(t), \ldots, \psi_{n}(t)\right)^{T}
$$

as a solution of the following differential system:

$$
\dot{\psi}=-A^{T} \psi, \quad \psi\left(t_{1}\right)=-\varphi^{\prime}(y) \text { for every } y \in \mathbb{R}^{n} .
$$

This system has the unique piecewise differentiable solution $\psi(t)=\psi(t, y)$ defined on $T$. Then a solution of problem (5.9) can be given by the following.

THEOREM 5.1 [17]. Let $\psi(t)=\psi(t, y), t \in T$ be a solution of conjugate system (5.11) for $y \in \mathbb{R}^{n}$. Then for an admissible control $z(t)=z(t, y)$ to be optimal in problem (5.11) it is necessary and sufficient that the condition $\langle\psi(t, y), B(t) z(t, y)\rangle=$ $\min _{u \in U}\langle\psi(t, y), B(t) u\rangle$ be fulfilled for almost every $t \in T$.

Using Theorem 5.1, we can easily show that the reachable set $\tilde{D}=\tilde{D}\left(t_{1}\right)$ is a simple set. Therefore, the value $\tilde{\pi}(\tilde{y})$ is calculated by the following scheme:

(1) Solve problem (5.11) for a given $\tilde{y} \in \mathbb{R}^{n}$. Let $\psi(t)=\psi(t, \tilde{y})$ be a solution of (5.11).

(2) Find the optimal control $z=z(t, \tilde{y})$ as a solution of the problem

$$
\langle\psi(t), B(t) u\rangle \longrightarrow \min , \quad u \in U
$$

at each moment of $t \in T$.

(3) Find a solution $\tilde{x}=x(t, z)$ of problem (5.2) for $u=z(t, \tilde{y})$.

(4) Find $\tilde{x}\left(t_{1}\right)=\tilde{x}\left(t_{1}, z\right)$ by the formula (5.4) for $t=t_{1}$.

(5) Calculate $\tilde{\pi}(\tilde{y})$ by the formula

$$
\tilde{\pi}(\tilde{y})=\max _{x \in \tilde{D}}\left\langle\varphi^{\prime}(\tilde{y}), x-\tilde{y}\right\rangle=\left\langle\varphi^{\prime}(\tilde{y}), \tilde{x}\left(t_{1}\right)-\tilde{y}\right\rangle .
$$

Now, based on Algorithm 4.3, we are ready to present an algorithm for solving problem (5.1), (5.2), and (5.3).

\section{Algorithm 5.2}

STEP 1. Let $k=0$ and an arbitrary control $u^{k} \in V$ be given.

Find $x^{k}=x\left(t_{1}, u^{k}\right)$ by solving the system (5.2) for $u=u^{k}$.

STEP 2. Solve the problem $\tilde{\pi}(y) \rightarrow \max , \varphi(y)=\varphi\left(x^{k}\right)$. Let $y_{k}$ be the solution of this problem and $z^{k}=z^{k}\left(t, y_{k}\right)$ be the solution of the corresponding problem

$$
\left\langle\varphi^{\prime}\left(y_{k}\right), x\right\rangle \longrightarrow \max , \quad x \in \tilde{D},
$$


or

$$
\left\langle\psi^{k}(t), B(t) z^{k}\right\rangle=\min _{u \in U}\left\langle\psi^{k}(t), B(t) u\right\rangle, \quad t \in T,
$$

where

$$
\dot{\psi}^{k}(t)=-A^{T}(t) \psi^{k}(t), \quad \psi^{k}\left(t_{1}\right)=-\varphi^{\prime}\left(y_{k}\right) .
$$

STEP 3. If $\pi\left(y^{k}\right) \leq 0$ then go to Step 5. Otherwise, go to Step 4 .

STEP 4. Set $u^{k+1}=z^{k}\left(t, y_{k}\right)$ and $k:=k+1$. Then go to Step 1 .

STEP 5. Iteration is terminated. $u^{*}=u^{k}$ is a global optimal solution of problem (5.1), (5.2), and (5.3).

Convergence of the algorithm is based on the following statement.

THEOREM 5.3. The sequence $\left\{u^{k}\right\} \subset V$ constructed by the Algorithm 5.2 is a maximizing sequence for problem (5.1), (5.2), and (5.3), i.e.,

$$
\lim _{k \rightarrow \infty} J\left(u^{k}\right)=\max _{u \in V} J(u)
$$

The proof is analogous to the method used in Theorem 4.2.

6. Numerical experiments. To check the efficiency of the algorithm proposed above, a few optimal control test problems have been considered. The proposed algorithm has been implemented on IBM PC/486 microcomputer in Pascal 7. The list of the test problems considered is the following:

$$
\begin{aligned}
& x_{1}^{2}(1)+x_{2}^{2}(1) \rightarrow \max , \\
& \dot{x}_{1}=u_{1}, \quad \dot{x}_{2}=u_{2}, \\
& x_{1}(0)=x_{2}(0)=0, \quad u(t) \in U, \quad t \in[0,1], \\
& U=\left\{u \in \mathbb{R}^{2} \mid-3 \leq u_{1} \leq 4,-2 \leq u_{2} \leq 5\right\}, \\
& x_{1}^{2}(1)+x_{2}^{2}(1) \rightarrow \max , \\
& \dot{x}_{1}=x_{2}+u_{1}, \quad \dot{x}_{2}=x_{1}+u_{2}, \\
& x_{1}(0)=x_{2}(0)=0, \quad u(t) \in U, \quad t \in[0,1], \\
& U=\left\{u \in \mathbb{R}^{2} \mid-3 \leq u_{i} \leq 4, i=1,2\right\}, \\
& x_{1}^{2}\left(t_{1}\right)+x_{2}^{2}\left(t_{1}\right)+x_{3}^{2}\left(t_{1}\right) \rightarrow \max , \\
& x_{1}=-x_{1}+x_{2}, \quad \dot{x}_{3}=x_{2}, \\
& \dot{x}_{2}=-x_{1}-x_{2}-10 u, \quad T=[0,2.8], \\
& x_{1}(0)=x_{2}(0)=0, \quad x_{3}(0)=-30, \\
& u(t) \in U=\{u \in \mathbb{R} \mid-15 \leq u \leq 15\} .
\end{aligned}
$$

The results of the numerical experiments for these problems are shown in Table 6.1. 
TABLE 6.1.

\begin{tabular}{lccc}
\hline Problems & Local value of $\varphi$ & Global value of $\varphi$ & Computing time (min:sec) \\
\hline 6.1 & 13 & 41 & $0: 51.00$ \\
\hline 6.2 & 53.1448 & 94.4798 & $0: 56.00$ \\
\hline 6.3 & 149.055 & 263.519 & $0: 06.78$ \\
\hline
\end{tabular}

7. Conclusions. In this paper, we considered some classes of global optimization problems including optimal control problem.

(1) Based on global optimality condition an algorithm for the solution of the problem of maximizing a convex function on a so-called "Simple" set has been proposed.

(2) The proposed algorithm was generalized to the nonconvex optimal control problem for a terminal functional.

(3) The proposed algorithm is shown to be convergent and was tested numerically on a few nonconvex optimal control problems.

ACKNOwledgements. I am indebted to Professors A. S. Strekalovsky and O. V. Vasillev for their encouragement and useful discussions on this paper. The author would like to thank Dr. M. Kamada and Dr. J. Simpson for their helpful advice.

\section{REFERENCES}

[1] R. E. Bellman and S. E. Dreyfus, Applied Dynamic Programming, Princeton University Press, Princeton, NJ, 1962. MR 25\#3791. Zbl 106.34901.

[2] R. Enkhbat, An algorithm for maximizing a convex function over a simple set, J. Global Optim. 8 (1996), no. 4, 379-391. MR 97i:90053. Zbl 851.90091.

[3] J. B. Hiriart-Urruty, From convex optimization to nonconvex optimization. Necessary and sufficient conditions for global optimality, Nonsmooth Optimization and Related Topics (Erice, 1988), Plenum, New York, 1989, pp. 219-239. MR 91a:90133. Zbl 735.90056.

[4] Hoang-Tuĭ, Concave programming with linear constraints, Dokl. Akad. Nauk SSSR 159 (1964), 32-35 (Russian). MR 31\#5694. Zbl 132.40103.

[5] R. Horst, On the global minimization of concave functions. Introduction and survey, Or Spektrum 6 (1984), no. 4, 195-205. MR 86d:90111. Zbl 551.65043.

[6] R. Horst and H. Tuy, Global Optimization, Springer-Verlag, Berlin, 1990, Deterministic Approaches. MR 92d:90002. Zbl 704.90057.

[7] A. D. Ioffe and V. M. Tihomirov, Theory of Extremal Problems, North-Holland Publishing Co., Amsterdam, 1979. MR 80d:49001b. Zbl 407.90051.

[8] Krotov, V. F. and Gurman, V. I., Metody i Zadachi Optimal Nogo Upravleniya. [Methods and Problems of Optimal Control], Izdat. "Nauka", Moscow, 1973. MR 54\#5931. Zbl 271.49003.

[9] B. M. Mukhamediev, Approximate method of solution of a concave programming problem, Zh. Vychisl. Mat. i Mat. Fiz. 22 (1982), no. 3, 727-732, 767. MR 83i:90123. Zbl 495.90065.

[10] P. M. Pardalos and J. B. Rosen, Constrained Global Optimization: Algorithms and Applications, Lecture Notes in Computer Science, 268, Springer-Verlag, Berlin, 1987. MR 89b:90191. Zbl 638.90064.

[11] L. S. Pontryagin, V. G. Boltyanskii, R. V. Gamkrelidze, and E. F. Mishchenko, The Mathematical Theory of Optimal Processes, Interscience Publishers John Wiley \& Sons, Inc. New York-London, 1962. MR 29\#3316b. Zbl 102.32001. 
[12] J. B. Rosen and P. M. Pardalos, Global minimization of large-scale constrained concave quadratic problems by separable programming, Math. Programming 34 (1986), no. 2, 163-174. MR 88i:90140. Zbl 597.90066.

[13] A. S. Strekalovsky, On the problem of Global Extremum, Soviet Math. Doklady of Science Academy 35 (1987), 194-198.

[14] - On global maximum of a convex terminal functional in optimal control problems, J. Global Optim. 7 (1995), no. 1, 75-91. MR 96f:49027. Zbl 843.49011.

[15] A. S. Strekalovsky and I. Vasiliev, On global search for non-convex optimal control problems, Developments in global optimization (Szeged, 1995), Kluwer Acad. Publ., Dordrecht, 1997, pp. 121-133. CMP 1483 361. Zbl 897.49016.

[16] F. P. Vasilev, Chislennye Metody Resheniya èkstremal Nykh Zadach. [Numerical Methods for Solving Extremal Problems], "Nauka", Moscow, 1988. MR 90g:65005. Zbl 661.65055 .

[17] O. V. Vasilev, Metody optimizatsii v funktsional nykh prostranstvakh. [Methods of Optimization in Function Spaces], Irkutsk. Gos. Univ., Irkutsk, 1979. MR 83f:49003. Zbl 513.90050 .

EnkHbat: Department of Mathematics, Mongolian State University, P.O. Box 46/635, MONGOLIA 


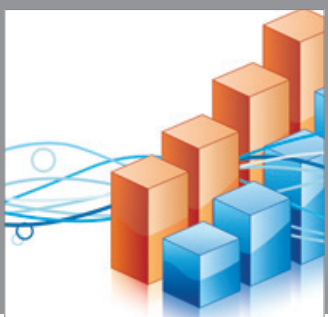

Advances in

Operations Research

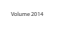

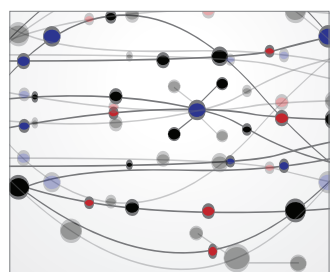

\section{The Scientific} World Journal
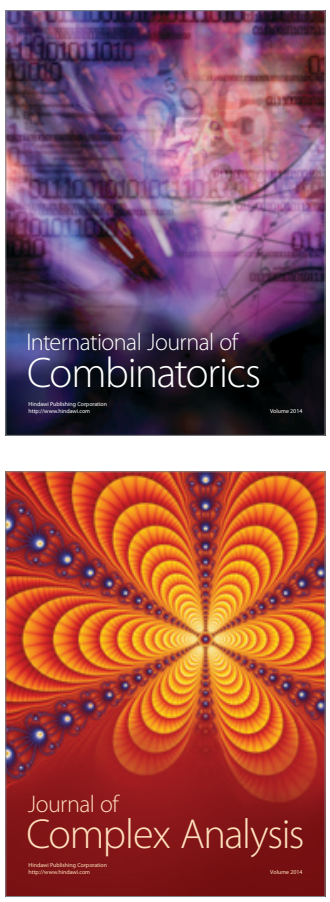

International Journal of

Mathematics and

Mathematical

Sciences
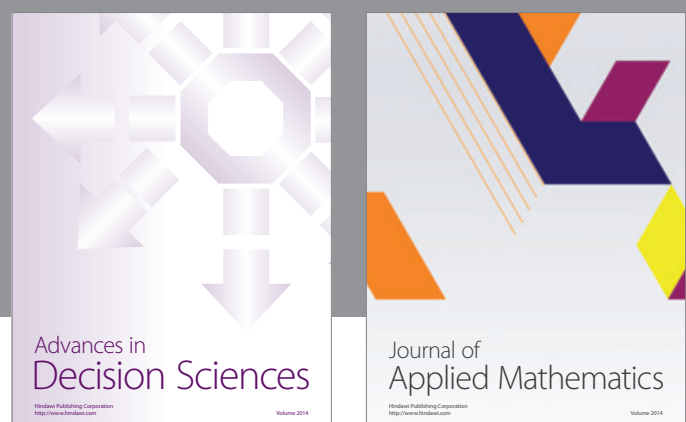

Journal of

Applied Mathematics
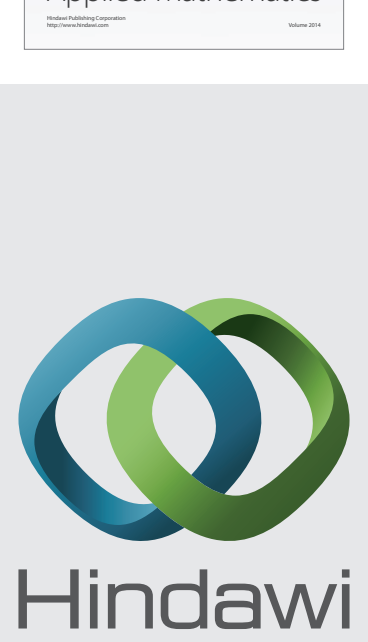

Submit your manuscripts at http://www.hindawi.com
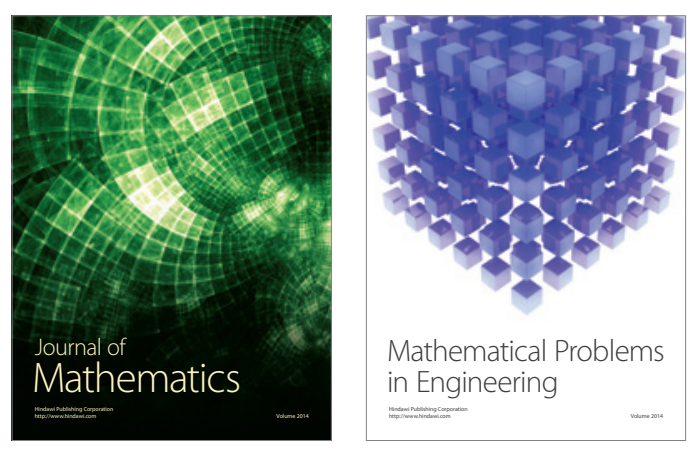

Mathematical Problems in Engineering
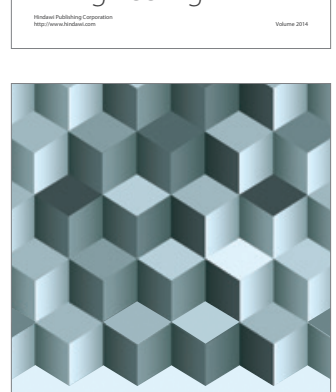

Journal of

Function Spaces
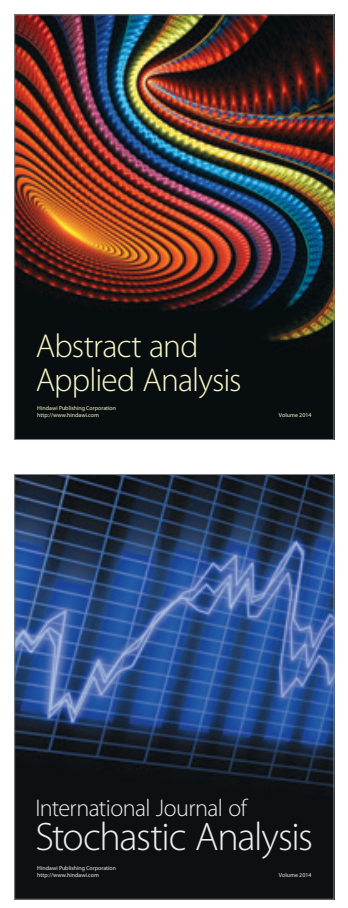

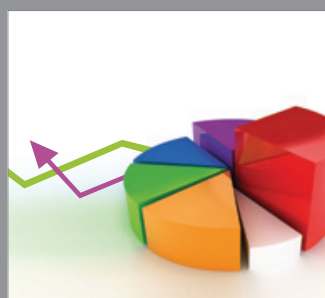

ournal of

Probability and Statistics

Promensencen
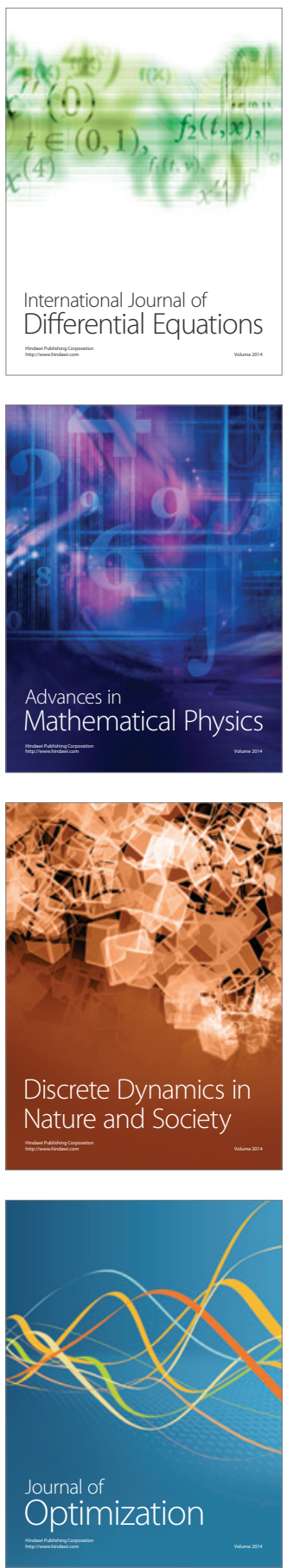